\title{
A MULTISCALE FINITE ELEMENT APPROACH FOR BUCKLING ANALYSIS OF ELASTOPLASTIC LONG FIBER COMPOSITES
}

\author{
Saeid Nezamabadi ${ }^{1}$, Hamid Zahrouni ${ }^{1}$, Julien Yvonnet $^{2}$ \\ $\&$ Michel Potier-Ferry ${ }^{1}$ \\ ${ }^{1}$ Université Paul Verlaine de Metz, Laboratoire de Physique et Mécanique des Matériaux, FRE \\ CNRS 3236, Ile du Saulcy 57045, Metz Cedex 01 France \\ ${ }^{2}$ Université Paris-Est, Laboratoire Modélisation et Simulation Multi Echelle, FRE CNRS 3160, 5 \\ Bd Descartes, 77454 Marne-la-Vallée Cedex 2, France
}

The present work is devoted to the microbuckling analysis of long fiber composites. A multiscale finite element method (FE2) is combined with the asymptotic numerical method (ANM) to study the elastoplastic instability which may occur in structures at both macroscopic and microscopic scales. The fiber is described by a linear material constitutive law, while the matrix phase is described by a nonlinear Ramberg-Osgood relationship. The stress field is then obtained via the total mechanical strain without any history dependence. Large strains are considered, which induce geometrical nonlinearities in both cases. The ANM framework allows obtaining complex response curves involving limit points in loading and displacement to be obtained. In the present path following procedure, adjustment of the step length is naturally automatic because the validity range of the asymptotic solution is a posteriori estimated depending on the local nonlinearity of the response branches. Numerical examples show the effectiveness of the proposed approach by investigating microscopic and macroscopic instabilities of long fiber composite structures in compression.

KEY WORDS: asymptotic numerical method, nonlinear homogenization, multiscale finite element method, long fiber composite, plastic microbuckling

\section{INTRODUCTION}

The development of numerical multiscale analysis has made possible new studies about effective properties of heterogeneous nonlinear materials containing dispersions of multiple phases in the microstructure. Thus, one alternative to meshing the whole structure, including heterogeneities, is to use a multiscale finite element procedure, also called in the literature computational homogenization, or nested finite element procedures (see, among many others, Smit et al., 1998; Feyel and Chaboche, 2000). In this framework, a nonlinear finite element problem is introduced under each material point (for example, integration points) to obtain the macroscopic stresssstrain relationship, without restriction on the deforma- tion level or the local nonlinear constitutive laws. Each local nonlinear microscopic problem is associated to a representative volume element (RVE). The macroscopic stresses are then obtained by direct averaging of the microscopic stresses, found by solving the local problem (Terada and Kikuchi, 2001; Ghosh et al., 2001; Feyel, 2003; Yvonnet and He, 2007).

Nevertheless, modeling multiscale buckling of heterogeneous materials remains a challenging task. The instability phenomena in the framework of multiscale homogenization analysis of heterogeneous materials are of structural and/or material types. These phenomena can occur on both macroscopic as well as microscopic scales and may influence each other. Abeyaratne and Triantafyllidis (1984) have investigated for the first time the instability 
phenomena in the heterogeneous materials. They studied the overall behavior of porous elastic media where they found that the homogenized material looses ellipticity, although the matrix material remains elliptic. Afterward, the instability of the heterogeneous materials originated numerous papers. (e.g., Triantafyllidis and Maker, 1985; Geymonat et al., 1993; Ohno et al., 2002; Okumura et al., 2002; Miehe et al., 2002; Gong et al., 2005; LopezPamies and Ponte Castañeda, 2005; deBotton et al., 2006; Michel et al., 2007; Yvonnet et al., 2007; Okumura et al., 2008).

Fiber microbuckling is one of the most famous instability phenomena in heterogeneous materials. It is one of the main mechanisms of long fiber composite compressive failure. Rosen (1964) presented the first approach of this type and showed that in an elastic context, microbuckling stress is approximately equal to the composite shear modulus. As established by Budiansky (1983), matrix physical nonlinearity and fiber initial waviness have to be considered to correctly predict the failure level. One can find many finite element microbuckling studies in the literature, for instance, Kyriakides et al. (1995) and Lee and Waas (1999). In Drapier et al. (1998, 1999, 2001), a multiscale approach is considered, but the microscopic model is solved analytically and the assumption of periodicity along the fiber is a bit restrictive. However, to our best knowledge, this paper represents the first attempt to model microbuckling by a multiscale finite element method, which is of fundamental interest for structure analysis of fiber composites. In the present paper, we study the plastic microbuckling of long fiber composites using multiscale finite element analysis $\left(\mathrm{FE}^{2}\right)$ combined with an asymptotic numerical method (ANM). We consider an initial fiber curvature and a nonlinear behavior of the matrix by choosing a Ramberg-Osgood relation (Chen and Han, 1998). The ANM is based on the expansion of nonlinear problems in the form of power series that are truncated at rather large orders. This allows one to obtain a series of linear problems. Consequently, within the framework of homogenization, one can construct the localization tensors using the superposition principal. These tensors are the same for all the linear problems deduced from the perturbation procedure. Hence, an explicit constitutive relation at the macroscopic level is computed for each asymptotic step. Furthermore, the length of steps is naturally adaptive and is estimated a posteriori using the previously computed terms of the series. This is not the case for the classical iterative algorithms based on Newton-Raphson procedure. This advantage allows one to follow complex response curves.
The layout of this paper is as follows: In Section 2, we present the variational formulations and the coupling equations between the macroscopic and microscopic problems. We also show how to adapt a nonlinear constitutive law to the ANM framework. In Section 3 details of the perturbation procedure applied to the multiscale problem are given. Resolution and path-following strategies are also presented. In Section 4 we present two main numerical examples involving instability phenomena in the long fiber-reinforced composites.

\section{MACROSCOPIC AND MICROSCOPIC VARIATIONAL FORMULATIONS}

\subsection{Equilibrium and Coupling Equations for Micro and Macro Problems}

We present in this section the variational equations needed to model the macroscopic and the periodic microscopic problems. Similar formulations have been presented in a previous paper (Nezamabadi et al., 2009) in which we considered a heterogeneous material involving large displacement and an elastic and linear constitutive law for the different phases. In the present paper we deal with similar problems but taking into account the plasticity behavior of the matrix at the microscopic level.

Let $\Omega$ ba a domain in $\mathbb{R}^{d}, d$ being the space dimension associated with a macroscopic structure and $\partial \Omega$ its external boundary, both in their reference configuration. In the framework of a total Lagrangian formulation, the weak form of the macroscopic equilibrium equation can be written as follows:

Find $\overline{\mathbf{u}} \in \mathcal{S}(\Omega)$ satisfying the essential boundary conditions, i.e. $\overline{\mathbf{u}}=\tilde{\mathbf{u}}$ on $\partial \Omega_{u}$, with $\tilde{\mathbf{u}}$ being the prescribed displacements and essential boundary, and $\mathcal{S}(\Omega)$ the space of sufficiently smooth functions, such that

$$
\int_{\Omega} \overline{\mathbf{P}}: \delta \overline{\mathbf{F}} d \Omega=\lambda T_{e x t}(\boldsymbol{\delta} \overline{\mathbf{u}}) \text { in } \Omega
$$

where the notation $(\overline{.})$ denotes the macroscopic quantities, $\overline{\mathbf{P}}$ is the first Piola-Kirchhoff tensor associated with a point $\overline{\mathbf{X}}$ of the macroscopic structure in its initial configuration, $\overline{\mathbf{u}}$ denotes the macroscopic displacement field, $\overline{\mathbf{F}}=\nabla_{\bar{X}} \overline{\mathbf{u}}+\mathbf{I}$ is the macroscopic deformation gradient tensor, and $\mathbf{I}$ is the second-order identity tensor. The term $T_{\text {ext }}(\delta \overline{\mathbf{u}})$ represents the virtual work of external load, $\lambda$ is a loading parameter, and $\delta \overline{\mathbf{u}} \in \mathcal{S}^{0}(\Omega), \mathcal{S}^{0}(\Omega)$ being the space of all displacement fields vanishing on $\partial \Omega_{u}$ and sufficiently smooth on $\Omega$. 
At this scale, the constitutive relationship between $\overline{\mathbf{P}}$ and $\overline{\mathbf{F}}$ is unknown. In the context of multiscale finite element analysis, the macroscopic stresses are obtained by solving a local nonlinear finite element problem related with the periodic microstructure.

For the microscopic scale, the material is assumed to be heterogeneous with a periodic microstructure, characterized by a representative volume element (RVE) that occupies a domain $\omega \in \mathbb{R}^{d}$ in its reference configuration. Let $\mathbf{u}(\mathbf{X})$ be a displacement at a microscopic point $\mathbf{X}$ in the undeformed configuration. The weak form associated with the microscopic problem is:

Find $\mathbf{u} \in \mathcal{S}(\omega)$ satisfying the microscopic boundary conditions, i.e., Eq. (5), such that

$$
\int_{\omega} \mathbf{P}: \delta \mathbf{F} d \omega=0 \text { in } \omega
$$

with $\delta \mathbf{u} \in \mathcal{S}^{0}(\omega), \mathcal{S}(\omega)$, and $S^{0}(\omega)$ being defined as previously. In (2), $\mathbf{P}$ and $\mathbf{F}$ denote, respectively, the first Piola-Kirchhoff stress tensor and the deformation gradient tensor at a microscopic point $\mathbf{X}$.

Problems (1) and (2) are coupled through two main relations. First, as the constitutive relation is not explicitly given at the macroscopic level, the effective stress tensor $\overline{\mathbf{P}}$ is obtained by considering an average value of the microscopic stress field over the RVE for a given point $\overline{\mathbf{X}}$ of the macroscopic structure. This relation is expressed as follows:

$$
\overline{\mathbf{P}}=\langle\mathbf{P}(\mathbf{X})\rangle=\frac{1}{|\omega|} \int_{\omega} \mathbf{P}(\mathbf{X}) d \omega,
$$

where $|\omega|$ represents the volume of the considered RVE.

The second relation concerns the mean value of the microscopic deformation gradient assumed in the form

$$
\overline{\mathbf{F}}=\langle\mathbf{F}(\mathbf{X})\rangle=\frac{1}{|\omega|} \int_{\omega} \mathbf{F}(\mathbf{X}) d \omega .
$$

This relation is deduced from the boundary conditions imposed on the RVE (Miehe, 2003). Generally, three main boundary conditions can be considered for the RVE as linear deformations, uniform tractions, or periodic constraints. In the proposed work, we consider periodic conditions on the boundary of the RVE, which can be recast into the following form:

$$
\mathbf{u}^{+}-\mathbf{u}^{-}=(\overline{\mathbf{F}}-\mathbf{I})\left(\mathbf{X}^{+}-\mathbf{X}^{-}\right) \text {on } \partial \boldsymbol{\omega},
$$

where $\mathbf{u}$ is the microscopic displacement, and the exponents + and - are associated with node indices on opposite sides of the RVE. Note that the boundary conditions depend on the macroscopic deformation tensor $\overline{\mathbf{F}}$.

\subsection{Microscopic Constitutive Relations}

At the microscopic scale, we assume that the constitutive relations are known in each phase of the RVE. For the applications that we target in the present work, a linear constitutive relation is considered for the fiber microconstituent and a nonlinear constitutive law is assumed for the matrix. In fact, we consider plastic behavior for the matrix without taking into account elastic unloading. Deformation theory of plasticity is used, which is convenient for problems where the physical nonlinearity is more important than the effect of irreversible process and the history of the loading (Hencky, 1924). Let us remark that Eq. (2) can be expressed in terms of the second Piola-Kirchhoff stress field $\mathbf{S}$ and the Green-Lagrange strain $\gamma$ as follows:

$$
\int_{\omega} \mathbf{S}: \delta \gamma d \omega=0
$$

The stress tensors $\mathbf{S}$ and $\mathbf{P}$ are related through $\mathbf{P}=$ FS and the Green-Lagrange strain is given by

$$
\begin{aligned}
\boldsymbol{\gamma} & =\frac{1}{2}\left(\mathbf{F}^{T} \mathbf{F}-\mathbf{1}\right)=\frac{1}{2}\left(\boldsymbol{\nabla}_{\mathbf{X}} \mathbf{u}+\boldsymbol{\nabla}_{\mathbf{X}} \mathbf{u}^{T}\right. \\
& \left.+\boldsymbol{\nabla}_{\mathbf{X}} \mathbf{u}^{T} \boldsymbol{\nabla}_{\mathbf{X}} \mathbf{u}\right)
\end{aligned}
$$

The linear constitutive relation for the fiber can be expressed as

$$
\mathrm{S}=\mathbb{C}: \gamma
$$

where $\mathbb{C}$ refers to the classical fourth-order elastic tensor. Concerning the matrix, we choose an elastoplastic constitutive law based on the Ramberg-Osgood relation (Chen and Han, 1998; Abaqus Theory Manual, 2008; Zahrouni et al., 1998), which is written in the 3D case in the following form:

$$
E \boldsymbol{\gamma}=(1+\boldsymbol{v}) \mathbf{S}^{d}-(1-2 \boldsymbol{v}) P \mathbf{I}+\frac{3}{2} \alpha\left[\frac{\mathbf{S}_{e q}}{\sigma_{y}}\right]^{n-1} \mathbf{S}^{d},
$$

where $E, \boldsymbol{v}, \alpha, n$, and $\sigma_{y}$ denote, respectively, the Young modulus, Poisson's ratio, yield offset, hardening component, and yield stress. $P=-1 / 3 \mathbf{S}: \mathbf{I}$ is the equivalent hydrostatic stress, $\mathbf{S}^{d}$ is the stress deviator defined by $\mathbf{S}^{d}=\mathbf{S}+P \mathbf{I}$. $\mathbf{S}_{e q}$ is the von-Mises equivalent stress defined as follows:

$$
\mathbf{S}_{e q}=\sqrt{\frac{3}{2} \mathbf{S}^{d}: \mathbf{S}^{d}} .
$$

As Eq. (9) is highly nonlinear, it is convenient to set it into a regular and quadratic form which is well desired in the framework of ANM (Potier-Ferry et al., 1997; 
Zahrouni et al., 1998; Abichou et al., 2002; Zahrouni et al., 2004). As the hardening exponent $n$ is not an integer, this law is not analytic for null stress. This is why a regularization procedure and a differential relation are introduced in this model, allowing expansions into power series (Zahrouni et al., 1998). For this purpose, the vonMises equivalent stress is slightly modified and recast in the following form:

$$
\mathbf{S}_{e q}^{2}=\frac{3}{2} \mathbf{S}^{d}: \mathbf{S}^{d}+\eta^{2} \sigma_{y}{ }^{2},
$$

where $\eta$ is a regularization parameter. For $\eta=0$, the initial constitutive law (9) is recovered. Furthermore, two additional variables $\mathrm{K}$ and $\zeta$ are introduced to transform the power law into a differential equation:

$$
\begin{aligned}
\kappa\left(\mathbf{S}_{e q}\right) & =\frac{3}{2} \alpha\left[\frac{\mathbf{S}_{e q}}{\sigma_{y}}\right]^{n-1} \\
& =\frac{3}{2} \alpha\left[\frac{3}{2 \sigma_{y}^{2}} \mathbf{S}^{d}: \mathbf{S}^{d}+\eta^{2}\right]^{\frac{n-1}{2}}, \\
\zeta^{2} & =\frac{\mathbf{S}_{e q}^{2}}{\sigma_{y}^{2}}=\frac{3}{2 \sigma_{y}^{2}} \mathbf{S}^{d}: \mathbf{S}^{d}+\eta^{2} .
\end{aligned}
$$

These two variables are linked as follows:

$$
\kappa=\frac{3}{2} \alpha \zeta^{n-1} .
$$

If we carry out a differentiation of Eq. (14), one obtains a relation which is more convenient for the asymptotic expansions:

$$
\zeta d \kappa=(n-1) \kappa d \zeta .
$$

In this way we obtain a general problem with a quadratic nonlinearity with respect to the new variables. Moreover, to keep the same initial slope as for nonregularized law, the first member of Eq. (9) is multiplied by $\left(1+\alpha \eta^{n}\right)$ (Zahrouni et al., 1998). The nonlinear constitutive law leads then to the five following equations:

$$
\begin{aligned}
& E\left(1+\alpha \eta^{n}\right) \gamma=(1+v) \mathbf{S}^{d}-(1-2 v) P I+\kappa \mathbf{S}^{d} \\
& \zeta^{2}=\frac{3}{2 \sigma_{y}^{2}} \mathbf{S}^{d}: \mathbf{S}^{d}+\eta^{2} \\
& \zeta d \kappa=(n-1) \kappa d \zeta \\
& \mathbf{S}^{d}=\mathbf{S}+P \mathbf{I} \\
& P=-\frac{1}{3} \mathbf{S}: \mathbf{I} .
\end{aligned}
$$

\section{ASYMPTOTIC NUMERICAL ALGORITHM}

The micro and macro formulations are described by the nonlinear system of Eqs. $(1,5-8,16)$. The regularization procedure and the differential form introduced in the nonlinear constitutive law have allowed a regular and quadratic framework to be obtained, which is preferred using ANM algorithms. The present section gives some details about the path following procedure within ANM. We show that the perturbation procedure allows one to transform the nonlinear problem into a sequence of linear ones. Because of coupling equations, at each asymptotic order, the solution of the resulting linear problems is obtained by using the superposition principal. Details of this procedure are given in Nezamabadi et al. (2009) and will be explained once more in what follows.

\subsection{Perturbation Technique}

(12) Solution of the nonlinear multiscale problem [Eqs. (1, 5$8,16)$ ] is sought using the asymptotic numerical method. This consists of expanding the main macroscopic and microscopic variables of this problem into a power series with respect to a path parameter. In this way, the nonlinear problem is transformed into a sequence of linear ones having the same tangent operator. If these variables are represented by a mixed vector $\Lambda=(\overline{\mathbf{u}}, \overline{\mathbf{P}}, \mathbf{u}, \mathbf{P}, \ldots)$, the perturbation technique applied to $\Lambda$ and to the load parameter $\lambda$ leads to

$$
\begin{gathered}
\boldsymbol{\Lambda}(a)=\boldsymbol{\Lambda}_{0}+\sum_{p=1}^{N} a^{p} \boldsymbol{\Lambda}_{p}, \\
\lambda(a)=\lambda_{0}+\sum_{p=1}^{N} a^{p} \lambda_{p},
\end{gathered}
$$

where $a$ is a path parameter to be defined, $N$ is the truncation order of the series, and $\Lambda_{0}$ and $\lambda_{0}$ denote a known initial solution.

By substituting Eqs. (17), (18) into (1, 5-8, 16), one obtains a sequence of linear problems. For example, for order $p$, Eq. (1) is written as:

$$
\int_{\Omega} \overline{\mathbf{P}}_{p}: \delta \overline{\mathbf{F}} d \Omega=\lambda_{p} T_{e x t}(\boldsymbol{\delta} \overline{\mathbf{u}}) .
$$

Concerning the microscopic variables, if we set the Green-Lagrange strain $\gamma$ into the following form:

$$
\begin{aligned}
\boldsymbol{\gamma} & =\frac{1}{2}\left(\boldsymbol{\nabla}_{\mathbf{X}} \mathbf{u}+\nabla_{\mathbf{X}} \mathbf{u}^{T}\right)+\frac{1}{2}\left(\nabla_{\mathbf{X}} \mathbf{u}^{T} \nabla_{\mathbf{X}} \mathbf{u}\right) \\
& =\gamma_{l}(\mathbf{u})+\gamma_{n l}(\mathbf{u}, \mathbf{u}),
\end{aligned}
$$


and

$$
\begin{aligned}
\delta \boldsymbol{\gamma} & =\boldsymbol{\gamma}_{l}(\boldsymbol{\delta} \mathbf{u})+\boldsymbol{\gamma}_{n l}(\boldsymbol{\delta} \mathbf{u}, \mathbf{u})+\gamma_{n l}(\mathbf{u}, \delta \mathbf{u}) \\
& =\boldsymbol{\gamma}_{l}(\boldsymbol{\delta} \mathbf{u})+\boldsymbol{\gamma}_{n l}^{s}(\mathbf{u}, \boldsymbol{\delta} \mathbf{u}) .
\end{aligned}
$$

Equation (6) can then be written for order $p$ as

$$
\begin{gathered}
\int_{\omega}\left(\mathbf{S}_{p}:\left(\boldsymbol{\gamma}_{l}(\boldsymbol{\delta} \mathbf{u})+\boldsymbol{\gamma}_{n l}^{s}\left(\mathbf{u}_{0}, \boldsymbol{\delta} \mathbf{u}\right)\right)\right. \\
\left.+\mathbf{S}_{0}: \boldsymbol{\gamma}_{n l}^{s}\left(\mathbf{u}_{p}, \boldsymbol{\delta} \mathbf{u}\right)\right) d \omega=0
\end{gathered}
$$

Boundary conditions at the microscopic level are expressed at order $p$ by

$$
\mathbf{u}_{p}^{+}-\mathbf{u}_{p}^{-}=\overline{\mathbf{F}}_{p}\left(\mathbf{X}^{+}-\mathbf{X}^{-}\right) \text {on } \partial \omega .
$$

Constitutive law is defined for each phase of the microscopic structure. In the case of the linear law (8), order $p$ leads to the following relation:

$$
\mathbf{S}_{p}=\mathbb{C}: \gamma_{p}
$$

and in the case of the nonlinear constitutive law (16), order $p$ gives

$$
\mathbf{S}_{p}=\mathbb{C}_{t}: \boldsymbol{\gamma}_{p}+\mathbf{S}_{p}^{\text {res }},
$$

where

$$
\boldsymbol{\gamma}_{p}=\boldsymbol{\gamma}_{l}\left(\mathbf{u}_{p}\right)+\boldsymbol{\gamma}_{n l}^{s}\left(\mathbf{u}_{0}, \mathbf{u}_{p}\right)+\sum_{i=1}^{p-1} \boldsymbol{\gamma}_{n l}\left(\mathbf{u}_{i}, \mathbf{u}_{p-i}\right),
$$

and $\mathbb{C}_{t}$ and $\mathbf{S}_{p}^{\text {res }}$ are defined in Appendix A.

\subsection{Resolution Strategy}

We have presented in Section 3.1 the perturbation technique which transforms the nonlinear problem to be solved into a sequence of linear ones. The aim of the present section is to give some details on how the resulting linear problems are solved in the context of a multiscale framework. Because of the coupling conditions between macroscopic and microscopic problems, we start by solving partly the linear problems at the microscopic level for each order. The obtained solution allows one to construct the localization tensor numerically and then to obtain the macroscopic tangent modulus. At this stage, one can solve the linear problem of the macroscopic equilibrium to obtain the displacement field $\bar{u}_{p}$. This displacement is used to compute the macroscopic deformation gradient $\overline{\mathbf{F}}_{p}$ and then to finish the computation of the variables at the microscopic level.
The linear problems at order $p$ of the microscopic level $(5,6,16)$ can be set in a generic form as follows:

$$
\begin{gathered}
\mathcal{L}\left(\mathbf{u}_{p}, \boldsymbol{\delta} \mathbf{u}\right)=\mathcal{F}_{p}^{n l}(\boldsymbol{\delta} \mathbf{u}) \text { in } \omega, \\
\mathbf{u}_{p}^{+}-\mathbf{u}_{p}^{-}=\overline{\mathbf{F}}_{p}\left(\mathbf{X}^{+}-\mathbf{X}^{-}\right) \text {on } \partial \omega .
\end{gathered}
$$

The operator $\mathcal{L}$ corresponds to the tangent stiffness of the microscopic structure. It is the same for all the linear problems resulting from the perturbation procedure. The right-hand side operator $\mathcal{F}_{p}^{n l}$ is given and depends only on the solutions of the previous linear problems $i(1 \leq i \leq$ $p$ ). Details about $\mathcal{L}$ and $\mathcal{F}_{p}^{n l}$ are given in Appendix B.

Note that the boundary conditions (28) depend on the local macroscopic deformation gradient, $\overline{\mathbf{F}}_{p}$, which is unknown. As the problems (27) are linear, we can use the superposition principle. Therefore, $\mathbf{u}_{p}$ can be expressed as a linear combination of the solution modes obtained by imposing independently homogeneous deformation fields (see Appendix C). The solution $\mathbf{u}_{p}$ can be then expressed in the form

$$
\mathbf{u}_{p}(\mathbf{X})=\mathbb{A}(\mathbf{X}): \overline{\mathbf{F}}_{p}+\mathbf{u}_{p}^{n l}(\mathbf{X})
$$

where $\mathbb{A}$ is a third-order tensor deduced from solutions of the linear problems obtained by the superposition principle, and $\mathbf{u}_{p}^{n l}$ is a vector associated with the right-hand term of Eq. (27).

Deriving (29) with respect to $\mathbf{X}$ and using $\nabla_{\mathbf{X}} \mathbf{u}_{p}=$ $\mathbf{F}_{p}$, we obtain

$$
\mathbf{F}_{p}=\mathbb{A}_{, \mathbf{X}}: \overline{\mathbf{F}}_{p}+\mathbf{u}_{p, \mathbf{X}}^{n l},
$$

where $\mathbb{A}_{, \mathbf{X}}$ is a fourth-order tensor, which is identified as a localization tensor, and $\mathbf{u}_{p, \mathbf{X}}^{n l}$ is a second-order tensor.

To achieve the homogenized relationship, we consider the asymptotic expansion of $\mathbf{P}=\mathbf{F S}$. After some elementary operations, one obtains at order $p$ the following relation:

$$
\mathbf{P}_{p}=\mathbb{L}: \overline{\mathbf{F}}_{p}+\mathbf{P}_{p}^{n l},
$$

where expressions of operators $\mathbb{L}$ and $\mathbf{P}_{p}^{n l}$ are detailed in Appendix D. The effective stresses can be then obtained at each order $p$ by averaging Eq. (31):

$$
\overline{\mathbf{P}}_{p}=\overline{\mathbb{L}}: \overline{\mathbf{F}}_{p}+\overline{\mathbf{P}}_{p}^{n l} \text {. }
$$

By introducing (32) into Eq. (19), we obtain the final form of the linear problems at order $p$ at the macroscopic level:

$$
\int_{\Omega} \overline{\mathbf{F}}_{p}: \overline{\mathbb{L}}: \delta \overline{\mathbf{F}} d \Omega=\lambda_{p} T_{e x t}(\delta \overline{\mathbf{u}})-\int_{\Omega} \overline{\mathbf{P}}_{p}^{n l}: \delta \overline{\mathbf{F}} d \Omega .
$$

Solution $\bar{u}_{p}$ of Eqs. (33) and (29) are used to finish the computation of the variables at the microscopic level for each integration point of the macroscopic structure. 


\subsection{Path-Following Strategy}

The path-following strategy is of critical importance, mainly in the presence of instability phenomena. This is the case of the present work where instability may occur in both macroscopic and microscopic levels.

Nonlinear problems involving instability behavior are generally solved by using Newton-Raphson iterative algorithms associated with arc-length techniques to follow complex branches involving limit points in loading and displacement responses. To optimize the computation time, one has to use step-size adjustments. Indeed, for a fixed step size, a very small step length can lead to a high number of steps; however, a large step length allows reduction of the number of steps but can lead to divergence or to a very large number of iterations. Furthermore, the linear iterative algorithms converge sometimes to an unwanted path in the case of problems involving strong nonlinear solution response (Eriksson and Kouhia, 1995). The best strategy must use large step lengths in the regions with very smooth response and small step lengths in the regions with strong nonlinearity, as for limit points or quasi-bifurcation situations. In the general case, the best strategy is not obvious (Ramm, 1981; Riks, 1984; Wriggers et al., 1988; Eriksson, 1991; Crisfield, 1991; Battini, 2007; Kouhia, 2008).

In the framework of ANM, the power series allows one to obtain a large part of the solution branch by decomposing only one tangent stiffness matrix. This procedure can be considered as a high-order predictor which generally does not need any correction phase. As the series has convergence radius, a continuation procedure can be easily implemented, considering that the relative difference between the solutions of two consecutive orders $N$ and $N-1$ must remain small enough as compared to a critical value $\delta$. If we consider the discretized form of the displacement field, one can determine a validity range of the truncated series using the following approximation:

$$
a_{\max }=\left(\delta \frac{\left\|\overline{\mathbf{u}}_{1}\right\|}{\left\|\overline{\mathbf{u}}_{N}\right\|}\right)^{\frac{1}{N-1}},
$$

where $\delta$ is the accuracy parameter and $\|$. $\|$ refers to the norm of the vector.

In this way, the whole solution is obtained in a stepby-step manner considering each end step as the starting point for the next one. Furthermore, the step length is naturally adaptive, depending upon the local nonlinearity of the response curve, since it is a posteriori computed using the terms of the series. Details of these procedures are given in many references (Cochelin, 1994; Zahrouni et al., 1999; Cochelin et al., 2007). This technique to compute the validity range is not unique; Assidi et al. (2009) proposed a procedure which consists in computing the validity range for all the variables expanded into power series and keeps the smallest value of $a_{\max }$. This ensures that all the equations of the considered problem are solved with a high accuracy. To improve the validity range of the asymptotic solution, we propose that the power series representation be replaced by rational fractions named Padé approximants. Experience shows that this technique allows one to reduce the computation time by about $50 \%$. Details of this technique are not given in this work. Readers can refer to the following references: Najah et al., 1998; Elhage-Hussein et al., 2000; Lahmam et al., 2002; Boutyour et al., 2004; Aggoune et al., 2004; and Aggoune et al., 2006.

At last, to solve the resulting linear problems (33) and (27), we need to define a constraint equation as for the path-following technique proposed with the classical Newton-Raphson algorithms. The computation can be managed by imposing a force, a displacement, or a combination of both [i.e., Riks technique (Riks, 1984)]. In the present work, a simple procedure is chosen by considering the projection of the macroscopic displacement field onto its tangent:

$$
a=\left(\overline{\mathbf{u}}(a)-\overline{\mathbf{u}}_{0}\right) \cdot \overline{\mathbf{u}}_{1} .
$$

Note that Eq. (35), which uses the macroscopic variable $\overline{\mathbf{u}}$, can be replaced by anyone using local variables as the displacement vector of the discretized RVE considered at an integration point of the structure. We can also combine many variables which do not exhibit the same behavior during the macroscopic loading.

\section{NUMERICAL EXAMPLES}

The accuracy and the efficiency of the proposed technique were studied in the elastic framework in Nezamabadi et al. (2009). The influence of microbuckling of the fiber with the initial imperfection on the macrostructure is assessed here. For this purpose, first, the plastic microbuckling mechanism is described briefly. Afterward, the compression of a fiber-reinforced composite material with the elastoplastic nonlinear constitutive law in the matrix is considered. The proposed problem has been discretized using a two-dimensional finite element in the plane stress framework. The macrostructure has been meshed with four node quadrangular elements, whereas the microstructure has been meshed with nine node quadrangular elements. 
In the context of ANM, the truncation order $\mathrm{N}$ of the series and the accuracy parameter $\delta$, which allows limiting the length of each asymptotic step in a continuation procedure, are important. In all the tests presented here, we have solved the nonlinear problems using ANM with Padé approximants and $N=15$, but $\delta=10^{-6}$ for the first test and $\delta=10^{-8}$ for the second one.

\subsection{Plastic Microbuckling Mechanism}

In this part we focus on the plastic microbuckling mechanism of the long fiber-laminated composite. A bidimensional representation of a laminate is used (see Fig. 1), where $\mathbf{e}_{2}$ is the $90^{\circ}$ direction corresponding to the loading direction. The imperfection magnitude $\left(v_{0}\right)$ is constant through the thickness. This mechanism was studied in several papers (Kyriakides et al., 1995; Drapier et al., $1998,1999,2001)$. Here we wish to revisit this mechanism by considering a microstructure with one fiber using the periodic boundary conditions at microscopic scale under plane stress conditions. The imperfection wavelength of a fiber is equal to $200 . \pi \mu \mathrm{m}$. The mechanical properties employed are shown in Table 1 which corresponds to a T300/914 composite. The parameters of the nonlinear behavior of the matrix are deduced from the work presented in Wisnom (1991). The microstructure is de-

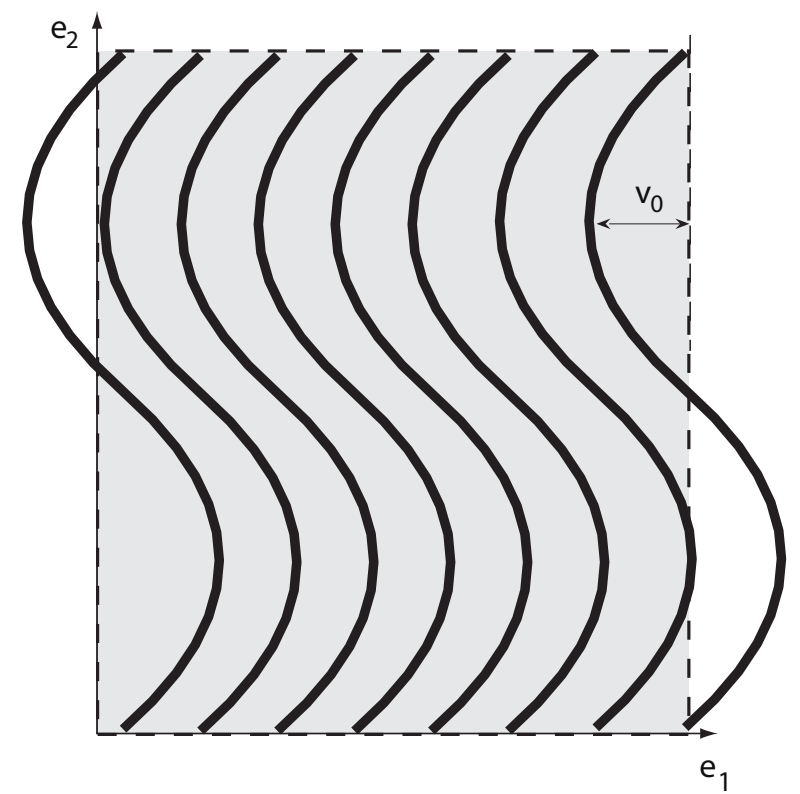

FIG. 1: The microstructure of long fiber composite with the imperfection magnitude.
TABLE 1: Mechanical characteristics for the T300/914 composite.

\begin{tabular}{|c|c|}
\hline $\begin{array}{c}\text { Fiber T300 } \\
\text { (isotropic) }\end{array}$ & $\begin{array}{c}\text { Matrix 914 } \\
\text { (isotropic) }\end{array}$ \\
\hline$E_{f}=240 \mathrm{Gpa}$ & $E_{m}=4.5 \mathrm{Gpa}$ \\
\hline$v_{f}=0.3$ & $v_{m}=0.4$ \\
\hline Fiber volume fraction: $f=0.6$ & $n=6$ \\
\hline Fiber diameter: $d_{f}=10 \mu \mathrm{m}$ & $\sigma_{y}=115 \mathrm{MPa}$ \\
\hline
\end{tabular}

formation driven by a macroscopic compression mode; $\overline{\mathbf{F}}-\mathbf{I}=\left[\bar{F}_{11}-1 ; \bar{F}_{12} ; \bar{F}_{21} ; \bar{F}_{22}-1\right]=[0 ; 0 ; 0 ;-1][$ see Eq. (5)].

Figure 2 shows the responses under compression mode for different imperfection magnitudes. These results are similar to ones obtained by Kyriakides et al. (1995). The stable and unstable responses toward prescribed strain can be distinguished. In the microstructure, the path following is well adopted and we can follow the response curve with a reasonable number of steps, despite the abrupt change of curvature at maximum stress.

\subsection{Interaction between Micro- and Macro- Instabilities}

To show the influence of plastic microbuckling on the macrostructure response, we consider the macrostructure shown in Fig. 3 which presents a rectangular plate clamped on three edges and submitted to a force distribution $\lambda P$ on the top edge. Because of the symmetry of the problem, only half of the structure is discretized. We use the same microstructure as for the previous example. The imperfection magnitude of fiber is $3 \mu \mathrm{m}$. We use our multiscale procedure to solve this micro-macro problem.

In Fig. 5, the displacement of a point located on the top of a macroscopic element shown in Fig. 4 versus the loading parameter is presented. This figure shows the influence of microscopic instability on the macroscopic one: initially, in the portion of the curve preceding point A, the macroscopic behavior is mainly linear. However, we can observe an abrupt change in the portion of the curve between point $\mathrm{A}$ and point $\mathrm{B}$. Note also the same phenomenon between the points $\mathrm{B}, \mathrm{C}, \mathrm{D}$, and $\mathrm{E}$. This is mainly caused by the occurrence of microscopic instabilities at different points. It is worth noting that thanks to the high order predictor of ANM, we can detect these instabilities. The deformed shapes of the microstructure at the integration point $I P I$ shown in Fig. 4 at points A, B , C, D, and $\mathrm{E}$ (see Fig. 5) are presented in Fig. 6. There is a sig- 


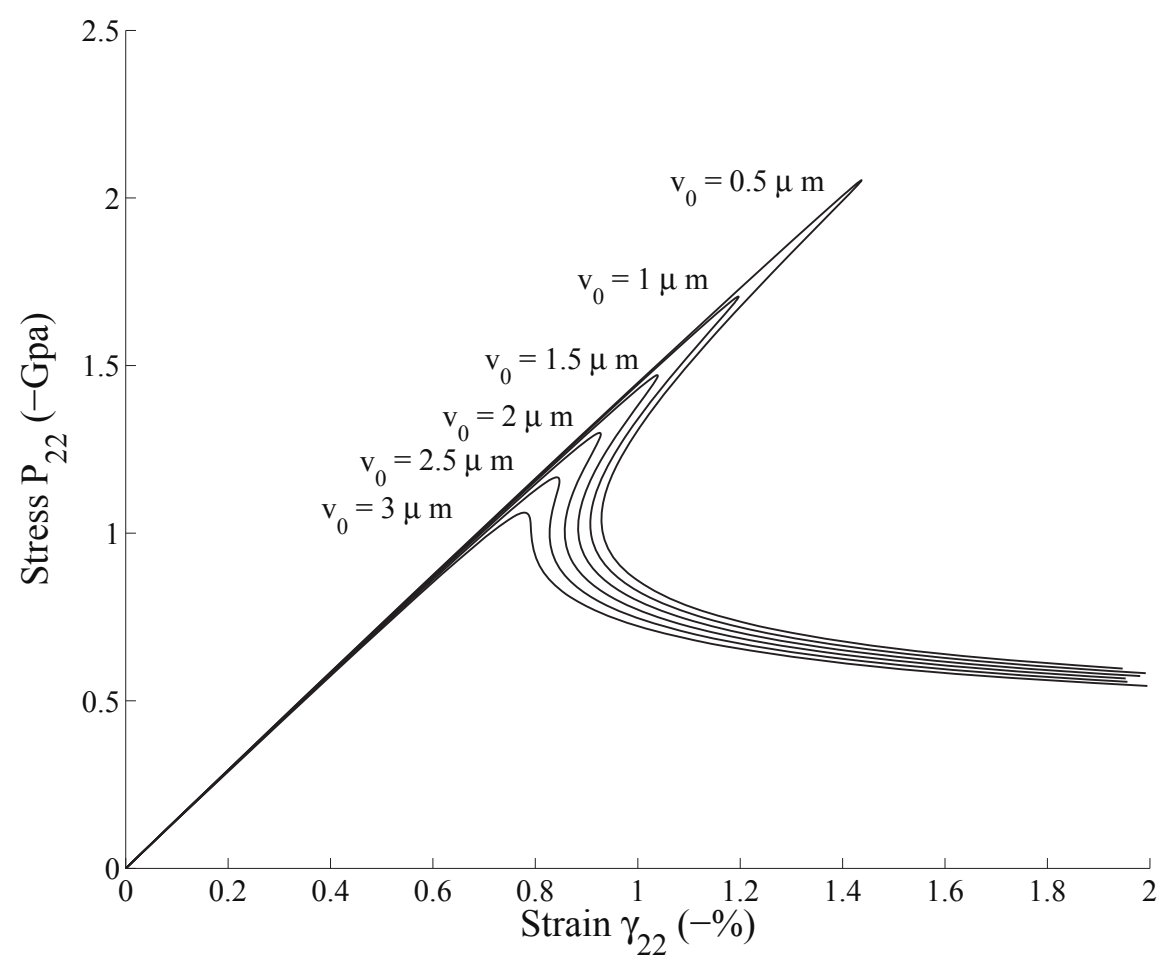

FIG. 2: Macroscopic stress-strain diagrams for the different imperfection magnitudes.

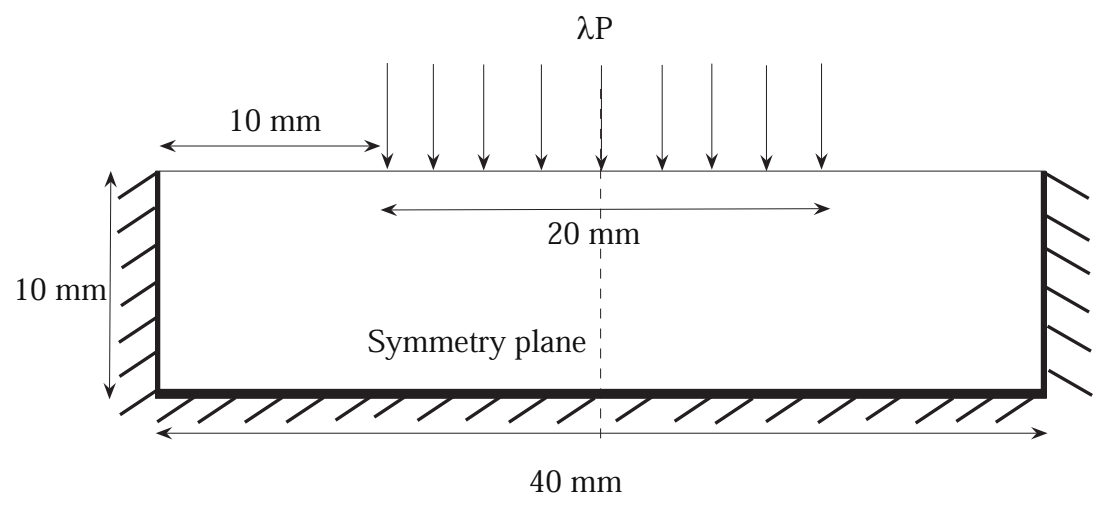

FIG. 3: Geometry and boundary conditions of the rectangular plate made of the fiber reinforced composites, $\mathrm{P}=1$ $N / m m$

nificant difference between these deformed microstructural shapes despite the small variation of macroscopic load, which illustrates the relationship between local and global instabilities. Furthermore, we observe that the microscopic buckling modes are the same for all points after the buckling of macrostructure $(\mathrm{B}, \mathrm{C}, \mathrm{D}$, and $\mathrm{E})$ which shows that we follow the same bifurcation branch.
In Fig. 7, the macroscopic stress-strain diagrams are presented for the different integration points shown in Fig. 4. The points $\mathrm{A}, \mathrm{B}, \mathrm{C}, \mathrm{D}$, and $\mathrm{E}$ in this figure are the same as in Fig. 5. Note that for the same magnitude of global load, the stresses at the integration points do not reach the same level. Hence, under compression mode, the different integration points do not pass the max- 


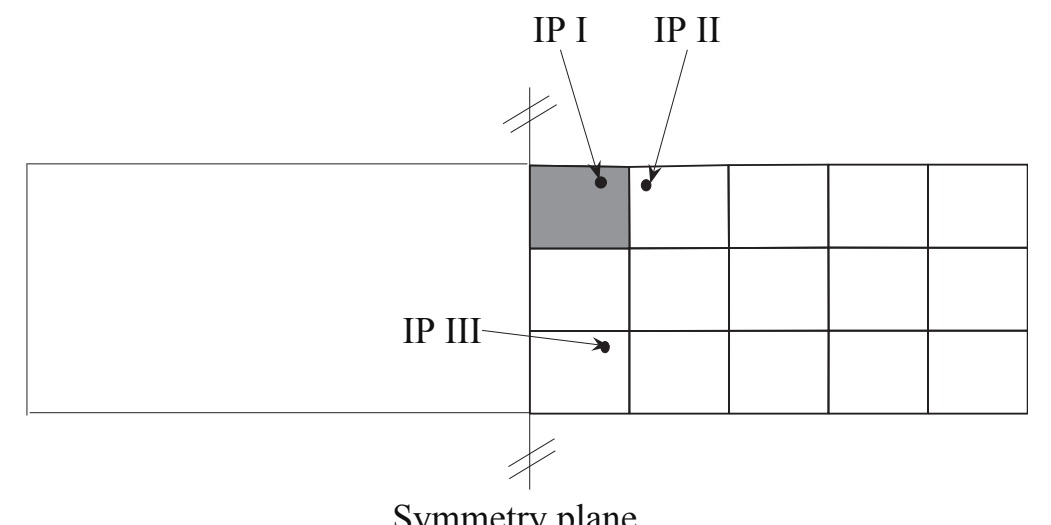

FIG. 4: Mesh topology of the macrostructure

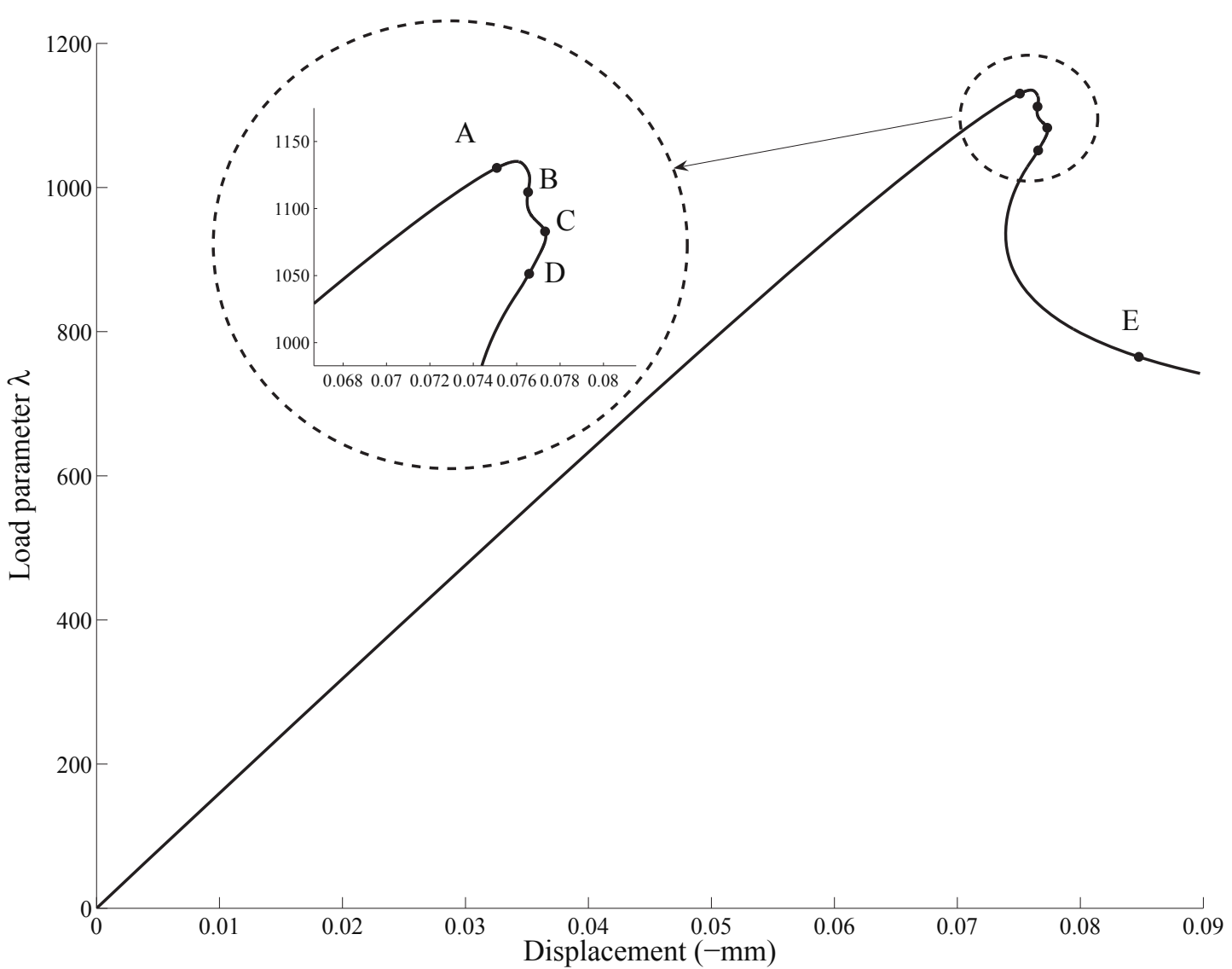

FIG. 5: The load-displacement diagram of the plastic microbuckling of fiber-reinforced composite problem. 


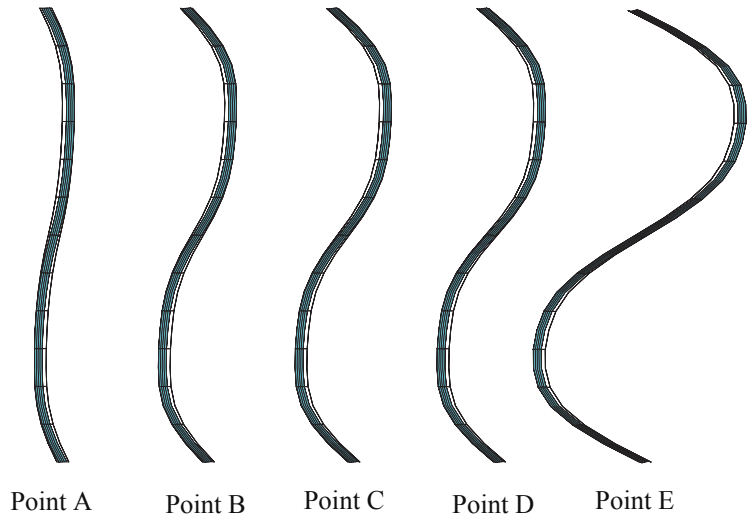

FIG. 6: Deformed shapes of microstructure at the integration point $I P I$ shown in Fig. 4 at different points presented in Fig. 5, deformation scale $=10$.

imum stress at the same time, which explains the shape of the load-displacement curve near the maximum load (see Fig. 7). This phenomenon consequentially induces a reduction of asymptotic step length. Nevertheless, ANM allows this type of response curves to be obtained, despite the appearance of successive instabilities.
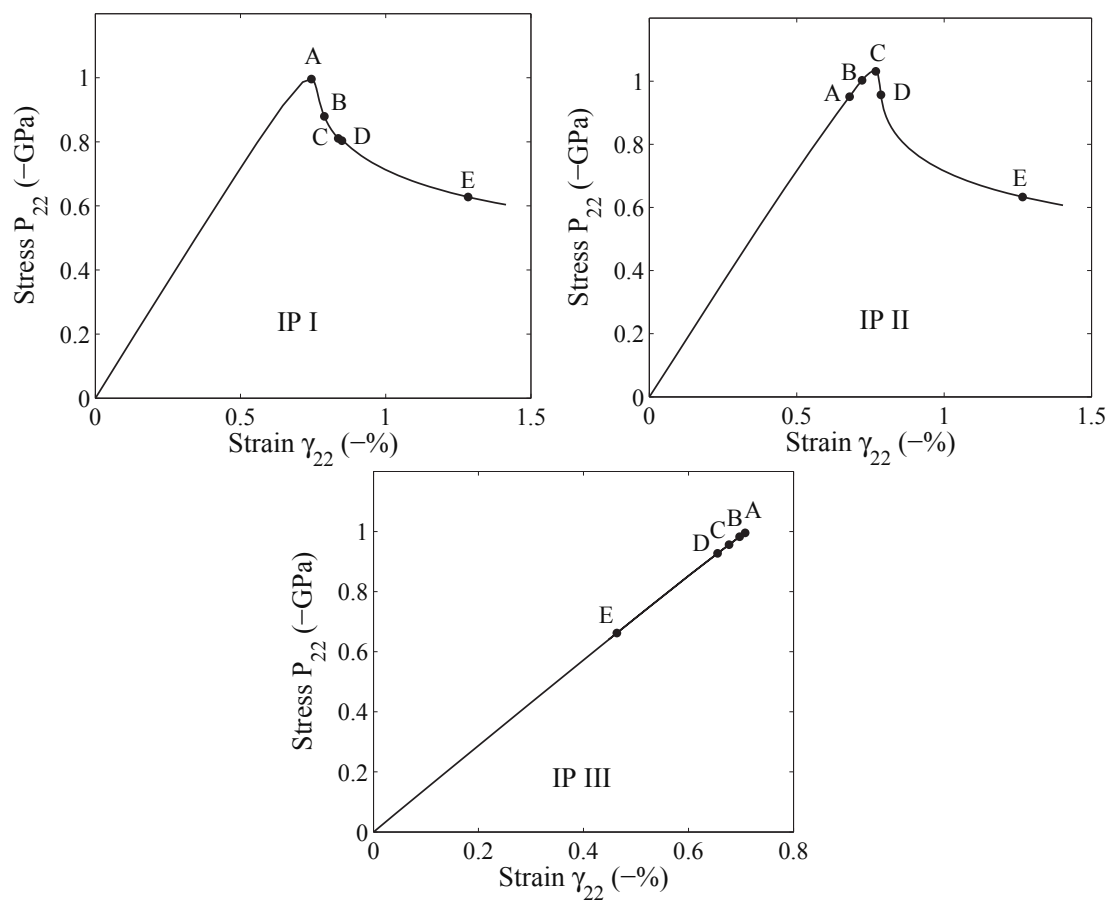

FIG. 7: The macroscopic stress-strain diagram for the integration points (I, II, and III) shown in Fig. 4. 
ered only one fiber with initial imperfection. We have then studied the compression of a fiber-reinforced composite with the microstructure defined above to assess the influence of the plastic microbuckling on the macrostructure behavior. The obtained results illustrate the capability of the proposed algorithm to deal with instabilities in the context of heterogeneous materials involving nonlinear constitutive relations. To our knowledge, a similar micro-macro analysis had never been proposed to account for the compressive failure of long fiber composites. Most of the numerical studies in the literature concern microscopic modeling that is more or less similar to that in Section 4.1 [see Kyriakides et al. (1995), and Lee and Waas (1999)]. In Drapier et al. (2001), the material properties were homogenized only transversally and not in the fiber direction, which permitted to account for structural effects across the thickness of a plate, but this analysis could not be coupled to any macro-structural problem, contrary to the one proposed in this paper.

However, accumulation of asymptotic steps is often observed which may be explained by the multiple instabilities and the loss of ellipticity close to the maximum load. To overcome this issue, one possible way of extension of the method is the use of second-order computational homogenization which allows obtaining in this context a well posed problem at the macroscopic level (Michel et al., 2007; Kouznetsova et al., 2004).

\section{REFERENCES}

Abaqus Theory Manual, Version 6.8, 2008, Hibbit, Karlsson and Sorensen Inc.

Abeyaratne, R. and Triantafyllidis, N., An investigation of localization in a porous elastic material using homogenization theory, J. Appl. Mech., vol. 51, pp. 481-486, 1984.

Abichou, H., Zahrouni, H., and Potier-Ferry, M., Asymptotic numerical method for problems coupling several nonlinearities, Comput. Methods Appl. Mech. Eng., vol. 191, pp. 57955810, 2002.

Aggoune, W., Zahrouni, H., and Potier-Ferry, M., High-order predictioncorrection algorithms for unilateral contact problems, J. Comput. Appl. Math., vol. 168, pp. 1-9, 2004.

Aggoune, W., Zahrouni, H., and Potier-Ferry, M., Asymptotic numerical methods for unilateral contact, Int. J. Numer. Method Eng., vol. 68, pp. 605-631, 2006.

Assidi, M., Zahrouni, H., Damil, N., and Potier-Ferry, M. Regularization and perturbation technique to solve plasticity problems, Int. J. Mater. Forming, vol. 2, pp. 1-14, 2009.

Battini, J. C., A modified corotational framework for triangu- lar shell elements, Comput. Methods Appl. Mech. Eng., vol. 196, pp. 1905-1914, 2007.

Budiansky, B., Micromechanics, Comput. Struct., vol. 16, pp. 3-12, 1983

Boutyour, E. H., Zahrouni, H., Potier-Ferry, M., and Boudi, M., Bifurcation points and bifurcated branches by an asymptotic numerical method and padé approximants, Int. J. Numer. Methods Eng., vol. 60, pp. 1987-2012, 2004.

Chen, W. F. and Han, D. J., Plasticity for Structural Engineers, Springer-Verlag, New York, 1998.

Cochelin, B., A path following technique via an asymptotic numerical method. Comput. Struct., vol. 53, pp. 1181-1192, 1994.

Cochelin, B., Damil, N., Potier-Ferry, M., Méthode Asymptotique Numérique, Hermès Science Publications, 2007.

Crisfield, M. A., Non-Linear Finite Element Analysis of Solids and Structures, Chichester: Wiley, vol. 1, 1991.

deBotton, G., Hariton, I., and Socolsky, E. A., Neo-Hookean fiber reinforced composites in finite elasticity, J. Mech. Phys. Solids, vol. 54, pp. 533-559, 2006.

Drapier, S., Grandidier, J. C., and Potier-Ferry, M., A nonlinear numerical approach to the analysis of microbuckling, Composites Sci. Technol., vol. 58, pp. 785-790, 1998.

Drapier, S., Grandidier, J. C., and Potier-Ferry, M., Towards a numerical model of the compressive strength for long fibre, European J. Mech. A-Solids, vol. 18, pp. 69-92, 1999.

Drapier, S., Grandidier, J. C., and Potier-Ferry, M., A structural approach of plastic microbuckling in long fibre composites: Comparison with theoretical and experimental results, Int. J. Solids Struct., vol. 38, pp. 3877-3904, 2001.

Elhage-Hussein, A., Potier-Ferry, M., and Damil, N., A numerical continuation method based on Padé approximants, Int. J. Solids Struct., vol. 37, pp. 6981-7001, 2000.

Eriksson, A., Derivatives of tangential stiffness matrices for equilibrium path descriptions, Int. J. Numer. Methods Eng., vol. 32, pp. 1093-1113, 1991.

Eriksson, A. and Kouhia, R., On step size adjustments in structural continuation problems, Comput. Struct., vol. 55, pp. 495-506, 1995.

Feyel, F. and Chaboche, J. L., $F E^{2}$ Multiscale approach for modelling the elastoviscoplastic behaviour of long fiber SiC/Ti composite materials, Comput. Methods Appl. Mech. Eng., vol. 183, pp. 309-330, 2000.

Feyel, F., A multilevel finite element method $\left(F E^{2}\right)$ to describe the response of highly non-linear structures using generalized continua, Comput. Methods Appl. Mech. Eng., vol. 192, pp. 3233-3244, 2003.

Geymonat, G., Muller, S., and Triantafyllidis, N., Homogenization of nonlinearly elastic materials: microscopic bifurcation and macroscopic loss of rank-one convexity, Arch. Ration. 
Mech. Anal., vol. 122, pp. 231-290, 1993.

Ghosh, S., Lee, K., and Raghavan, P., A multilevel computational model for multi-scale damage analysis in composite and porous media, Int. J. Solids Struct., vol. 38, pp. 2335$2385,2001$.

Gong, L., Kyriakides, S., and Triantafyllidis, N., On the stability of kelvin cell foams under compressive loads, J. Mech. Phys. Solids, vol. 53, pp. 771-794, 2005.

Hencky, H., Zur theorie deformationen und der hierdurch im material hervorgerufnen nebenspannungen, Zeitschrift Fr Angewandte mathematik und Mechanik, vol. 4, pp. 323-334, 1924.

Kouhia, R., Stabilized forms of orthogonal residual and constant incremental work control path following next term methods, Comput. Methods Appl. Mech. Eng., vol. 197, pp. 1389-1396, 2008.

Kousnetzova, V. G., Geers, M. G. $\quad$ D., and Brekelmans, W. A. M., Multi-scale second order computational homogenization pf multi-phase materials: A nested finite element solution strategy, Comput. Methods Appl. Mech. Eng., vol. 193, pp. 5525-5550, 2004.

Kyriakides, S., Arseculeratne, R., Perry, E. J., and Liechti, K. M., On the compressive failure of fiberreinforced composites, Int. J. Solids Struct., vol. 32, pp. 689-738, 1995.

Lahmam, H., Cadou, J. M., Zahrouni, H., Damil, N., and Potier-Ferry, M., High-order predictor-corrector algorithms, Int. J. Numer. Methods Eng., vol. 55, pp. 685-704, 2002.

Lee, S. H. and Waas, A. M., Compressive response and failure of fiber reinforced unidirectional composites, Int. J. Fracture, vol. 100, pp. 275-306, 1999.

Lopez-Pamies, O., Ponte Castañeda, P., Second-order estimated for the macroscopic response and loss of ellipticity in porous rubbers at large deformations, J. Elasticity, vol. 76, pp. 247-287, 2005.

Michel, J. C., Lopez-Pamies, O., Ponte Castanñeda, P., and Triantafyllidis, N., Microscopic and macroscopic instabilities in finitely strained porous elastomers, J. Mech. Phys. Solids, vol. 55, pp. 900-938, 2007.

Miehe, C., Schroder, J., and Becker, M., Computational homogenization analysis in finite elasticity: material and structural instabilities on the micro- and macro-scales of periodic composites and their interactions, Comput. Methods Appl. Mech. Eng., vol. 191, pp. 4971-5005, 2002.

Miehe, C., Computational micro-to-macro transitions for discretized micro-structures of heterogeneous materials at finite strains based on the minimization of averaged incremental energy, Comput. Methods Appl. Mech. Eng., vol. 192, pp. 559-591, 2003.

Najah, A., Cochelin, B., Damil, N., and Potier-Ferry, M., A critical review of asymptotic numerical methods, Arch.
Comput. Methods Eng., vol. 5, pp. 3-22, 1998.

Nezamabadi, S., Yvonnet, J., Zahrouni, H., and PotierFerry, M., A multilevel computational strategy for handling microscopic and macroscopic instabilities, Comput. Methods Appl. Mech. Eng., vol. 198, pp. 2099-2110, 2009.

Ohno, N., Okumura, D., and Noguchi, H., Microscopic symmetric bifurcation condition of cellular solids based on a homogenization theory of finite deformation, J. Mech. Phys. Solids, vol. 52, pp. 1125-1153, 2002.

Okumura, D., Ohno, N., and Noguchi, H., Post-buckling analysis of elastic honeycombs subject to in-plane biaxial compression, Int. J. Solids Struct., vol. 39, pp. 3487-3503, 2002.

Okumura, D., Okada, A., and Ohno, N., Buckling behaviour of

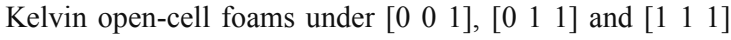
compressive loads, Int. J. Solids Struct., vol. 45, pp. 38073820, 2008.

Potier-Ferry, M., Damil, N., Braikat, B., Descamps, J., Cadou, J. M., Cao, H. L., and Elhage Hussein, A., Traitement des fortes non-linéarités par la méthode asymptotique numérique, Comptes Rendus de l'Académie des SciencesSeries IIB, vol. 324, pp. 171-177, 1997.

Ramm, E., Strategies for tracing the nonlinear response near limit points. In Nonlinear Finite Element Analysis in Struct. Mech., Wunderlish W, Stein E, Bathe KJ (eds), Springer: Berlin, pp. 63-89, 1981

Riks, E., Some computational aspects of the stability analysis of nonlinear structures, Comput. Methods Appl. Mech. Eng., vol. 47, pp. 219-259, 1984.

Rosen, B. W., Mechanics of composite strengthening, Fiber Composite Materials, American Society for Metals Seminar, vol. 3, pp. 37-75, 1964.

Smit, R., Brekelmans, W., Meijer, H., Prediction of the mechanical behaviour of nonlinear heterogeneous systems by multi-level finite element modelling, Comput. Methods Appl. Mech. Eng., vol. 155, pp. 181-192, 1998.

Terada, K. and Kikuchi, N., A class of general algorithms for multi-scale analysis of heterogeneous media, Comput. Methods Appl. Mech. Eng., vol. 190, pp. 5427-5464, 2001.

Triantafyllidis, N. and Maker, B. N., On the comparison between microscopic and macroscopic instability mechanisms in a class of fiber-reinforced composites, J. Appl. Mech., vol. 52, pp. 794-800, 1985.

Wriggers, P., Wagner, W., and Miehe, C., A quadratically convergent procedure for the calculation of stability point in finite element analysis, Comput. Methods Appl. Mech. Eng., vol. 70, pp. 329-347, 1988.

Yvonnet, J. and He, Q.-C., The reduced model multiscale method (R3M) for the non-linear homogenization of hyperelastic media at finite strains, J. Comput. Phys., vol. 223, pp. 341-368, 2007.

Yvonnet, J., Zahrouni, H., and Potier-Ferry, M., A model re- 
duction method for the post-buckling analysis of cellular microstructures, Comput. Methods Appl. Mech. Eng., vol. 197, pp. 265-280, 2007.

Wisnom, M. R., The effect of specimen size on the bending strength of unidirectional carbon fibre-epoxy, Composites Structures, vol. 18, pp. 47-63, 1991.

Zahrouni, H., Aggoune, W., Brunelot, J., and Potier-Ferry, M., Asymptotic numerical method for strong nonlinearities, $R e$ vue Européenne des Eléments Finis, vol. 118, pp. 13-97, 2004.

Zahrouni, H., Braikat, B., Damil, N., and Potier-Ferry, M., Solving plasticity problems by a perturbation technique, Proc. Appl. Math. Mech., vol. 5, pp. 455-456, 2005.

Zahrouni, H., Cochelin, B., and Potier-Ferry, M., Computing finite rotations of shells by an asymptotic-numerical method, Comput. Methods Appl. Mech. Eng., vol. 175, pp. 71-85, 1999.

Zahrouni, H., Potier-Ferry, M., Elasmar, H., and Damil, N., Asymptotic numerical method for nonlinear constitutive laws, Revue Européenne des Eléments Finis, vol. 7, pp. 841869, 1998.

\section{APPENDIX A.}

In the preceding sections, we have developed the asymptotic numerical algorithm for problems involving nonlinear constitutive laws. By comparison with the elastic case, we have replaced the elastic stiffness tensor $\mathbb{C}$ by the tangent modulus one $\mathbb{C}_{t}$ to compute the tangent stiffness matrix. Furthermore, because of this nonlinear law, stress field involves, at order $p$, residual vector $\mathbf{S}_{p}^{r e s}$. This latter leads to additional terms in the right hand side $\mathbf{F}_{p}^{n l}$. Hence, at order 1, we can write the constitutive relation in the following form:

$$
\mathbf{S}_{1}=\mathbb{C}_{t}: \gamma_{1}
$$

One can show easily that this equation is provided from the expression detailed below:

$$
\begin{gathered}
\mathbf{S}_{1}=C_{1} \gamma_{1}+C_{2}\left(\gamma_{1}: I\right) I+C_{3}\left(\gamma_{1}: \mathbf{S}_{0}^{d}\right) \mathbf{S}_{0}^{d}, \\
\mathbf{S}_{1}=\left[C_{1} \Im+C_{2} I \otimes I+C_{3} \mathbf{S}_{0}^{d} \otimes \mathbf{S}_{0}^{d}\right]: \gamma_{1}=\mathbb{C}_{t}: \gamma_{1},
\end{gathered}
$$

where $\Im$ is the fourth-order identity tensor and $C_{1}, C_{2}$ and $C_{3}$ are constants defined as follows:

$$
\begin{aligned}
C_{1} & =\frac{E\left(1+\alpha \eta^{n}\right)}{1+v+\kappa_{0}}, \\
C_{2} & =\frac{\left(3 v+\kappa_{0}\right) C_{1}}{3(1-2 v)},
\end{aligned}
$$

$$
C_{3}=\frac{-K C_{1}}{1+v+\mathrm{\kappa}_{0}+K \mathbf{S}_{0}^{d}: \mathbf{S}_{0}^{d}},
$$

where

$$
K=\frac{3(n-1) \kappa_{0}}{2 \sigma_{0}^{2} \zeta_{0}^{2}} .
$$

At order $p$, the constitutive relation is expressed as

$$
\mathbf{S}_{p}=\mathbb{C}_{t}: \boldsymbol{\gamma}_{p}+\mathbf{S}_{p}^{r e s} .
$$

Matrix $\mathbb{C}_{t}$ is the same at each order; however, residual stresses are expressed at order $p$ as follows:

$$
\begin{aligned}
\mathbf{S}_{p}^{r e s} & =\frac{1}{1+\boldsymbol{v}+\mathrm{\kappa}_{0}}\left\{C _ { 5 } \left[\vartheta \mathbf{S}_{0}^{d}: \mathbf{S}_{0}^{d}\right.\right. \\
& \left.\left.+\left(\sum_{r=1}^{p-1} \mathrm{\kappa}_{r} \mathbf{S}_{p-r}^{d}\right): \mathbf{S}_{0}^{d}\right]-\vartheta\right\} \mathbf{S}_{0}^{d} \\
& -\frac{1}{1+\boldsymbol{v}+\mathrm{\kappa}_{0}} \sum_{r=1}^{p-1} \kappa_{r} \mathbf{S}_{p-r}^{d}
\end{aligned}
$$

where

$$
\begin{gathered}
C_{5}=\frac{K}{1+\vartheta+\kappa_{0}+K \mathbf{S}_{0}^{d}: \mathbf{S}_{0}^{d}} \\
\vartheta=\sum_{r=1}^{p-1} \frac{(p-r)(n-1)-r}{p \zeta_{0}} \zeta_{p-r} \boldsymbol{\kappa}_{r} \\
+\frac{3(n-1) \kappa_{0}}{4 \sigma_{0}^{2} \zeta_{0}^{2}} \sum_{r=1}^{p-1} \mathbf{S}_{r}^{d}: \mathbf{S}_{p-r}^{d} \\
-\frac{(n-1) \kappa_{0}}{2 \zeta_{0}^{2}} \sum_{r=1}^{p-1} \zeta_{r} \zeta_{p-r} .
\end{gathered}
$$

\section{APPENDIX B.}

Expansion of (6) gives

$$
\int_{\omega}\left(\mathbf{S}_{0}+\sum_{p=1}^{N} a^{p} \mathbf{S}_{p}\right):\left(\boldsymbol{\delta} \boldsymbol{\gamma}_{0}+\sum_{p=1}^{N} a^{p} \boldsymbol{\delta} \boldsymbol{\gamma}_{p}\right) d \boldsymbol{\omega}=0
$$

The tensors $\delta \gamma_{0}$ and $\delta \gamma_{p}$ are given by expressing $\delta \gamma$ [Eq. (21)]:

$$
\begin{aligned}
\boldsymbol{\delta} \boldsymbol{\gamma}_{0} & =\frac{1}{2}\left(\boldsymbol{\nabla}_{X} \boldsymbol{\delta} \mathbf{u}+\boldsymbol{\nabla}_{X} \boldsymbol{\delta} \mathbf{u}^{T}+\boldsymbol{\nabla}_{X} \mathbf{u}_{0}^{T} \boldsymbol{\nabla}_{X} \boldsymbol{\delta} \mathbf{u}\right. \\
& \left.+\boldsymbol{\nabla}_{X} \boldsymbol{\delta} \mathbf{u}^{T} \boldsymbol{\nabla}_{X} \mathbf{u}_{0}\right)=\mathbb{B}: \boldsymbol{\nabla}_{X} \boldsymbol{\delta} \mathbf{u} \\
\boldsymbol{\delta} \boldsymbol{\gamma}_{p} & =\frac{1}{2}\left(\boldsymbol{\nabla}_{X} \mathbf{u}_{p}^{T} \boldsymbol{\nabla}_{X} \boldsymbol{\delta} \mathbf{u}+\boldsymbol{\nabla}_{X} \boldsymbol{\delta} \mathbf{u}^{T} \boldsymbol{\nabla}_{X} \mathbf{u}_{p}\right)
\end{aligned}
$$


where $\mathbb{B}$ is a fourth order tensor depending on the initial solution $\mathbf{u}_{0}$.

Now let us express $\mathbf{S}_{p}$ from equation (24) for the linear law and equation (25) for the nonlinear constitutive law. In these equations, we need to identifify $\gamma_{p}$. Hence, we expand the Green-Lagrange strain (20) that gives at order $p$ :

$$
\begin{aligned}
\boldsymbol{\gamma}_{p} & =\frac{1}{2}\left\{\boldsymbol{\nabla}_{X} \mathbf{u}_{p}+\boldsymbol{\nabla}_{X} \mathbf{u}_{p}^{T}+\boldsymbol{\nabla}_{X} \mathbf{u}_{0}^{T} \boldsymbol{\nabla}_{X} \mathbf{u}_{p}\right. \\
& \left.+\boldsymbol{\nabla}_{X} \mathbf{u}_{p}^{T} \boldsymbol{\nabla}_{X} \mathbf{u}_{0}\right\}+\boldsymbol{\gamma}_{p}^{n l},
\end{aligned}
$$

where

$$
\gamma_{1}^{n l}=0
$$

$$
\boldsymbol{\gamma}_{p}^{n l}=\frac{1}{2} \sum_{i=1}^{p-1} \boldsymbol{\nabla}_{X} \mathbf{u}_{i}^{T} \boldsymbol{\nabla}_{X} \mathbf{u}_{p-i}, \quad \forall p=2, \ldots, N .
$$

As $\gamma_{p}$ is linear with respect to $\nabla_{X} \mathbf{u}_{p}$, equation (B.4) can be rewritten in the form:

$$
\gamma_{p}=\mathbb{B}: \nabla_{X} \mathbf{u}_{p}+\gamma_{p}^{n l}=\mathbb{B}: \mathbf{F}_{p}+\gamma_{p}^{n l} .
$$

Then $\mathbf{S}_{p}$ is expressed by:

$$
\mathbf{S}_{p}=\mathbb{C}^{(r)}: \mathbb{B}: \mathbf{F}_{p}+\mathbf{S}_{p}^{n l},
$$

where $\mathbb{C}^{(r)}$ is equal to $\mathbb{C}$ for the linear constitutive law and $\mathbb{C}_{t}$ for the nonlinear relation, and the residual stress vector, $\mathbf{S}_{p}^{n l}$, is defined in the case of the linear law as:

$$
\mathbf{S}_{p}^{n l}=\mathbb{C}: \gamma_{p}^{n l},
$$

and in the case of the nonlinear constitutive law as:

$$
\mathbf{S}_{p}^{n l}=\mathbb{C}_{t}: \gamma_{p}^{n l}+\mathbf{S}_{p}^{r e s}
$$

By substituting Eq. (B.7) in (B.1), we obtain Eq. (27). The explicit forms of the operators involved in the resolution of the sequence of linear problems in equation (27) are given by:

$$
\mathcal{L}\left(\mathbf{u}_{p}, \boldsymbol{\delta} \mathbf{u}\right)=\int_{\Omega}\left\{\mathbf{S}_{0}: \delta \boldsymbol{\gamma}_{p}+\mathbf{F}_{p}: \mathbb{B}: \mathbb{C}^{(r)}: \delta \boldsymbol{\gamma}_{0}\right\} d \Omega
$$

and

$$
\mathcal{F}_{p}^{n l}(\boldsymbol{\delta} \mathbf{u})=-\int_{\Omega} \sum_{i=1}^{p-1}\left\{\mathbf{S}_{i}: \delta \boldsymbol{\gamma}_{p-i}+\mathbf{S}_{p}^{n l}: \delta \boldsymbol{\gamma}_{0}\right\} d \Omega
$$

\section{APPENDIX C.}

As each problem at order $p$ is linear, its solution $\mathbf{u}_{p}(\mathbf{X})$ can be decomposed into modes associated with different boundary condition responses. Here we give an illustration in the 2D case, though the methodology can be readily extended to three dimensions. We can write

$$
\begin{array}{r}
\mathbf{u}_{p}(\mathbf{X})=\mathbf{u}_{p}^{l}(\mathbf{X})+\mathbf{u}_{p}^{n l}(\mathbf{X}) \text { in } \omega, \\
\mathbf{u}_{p}^{l}(\mathbf{X})=\bar{F}_{p}^{11} \tilde{\mathbf{u}}^{(11)}(\mathbf{X})+\bar{F}_{p}^{12} \tilde{\mathbf{u}}^{(12)}(\mathbf{X}) \\
+\bar{F}_{p}^{21} \tilde{\mathbf{u}}^{(21)}(\mathbf{X})+\bar{F}_{p}^{22} \tilde{\mathbf{u}}^{(22)}(\mathbf{X}),
\end{array}
$$

where $\tilde{\mathbf{u}}^{(i j)}(\mathbf{X})$ are the solutions of the following problems:

$$
\left\{\begin{array}{l}
\mathcal{L}\left(\tilde{\mathbf{u}}^{(i j)}, \boldsymbol{\delta} \mathbf{u}\right)=0 \text { in } \omega, \\
\tilde{\mathbf{u}}^{(i j)+}-\tilde{\mathbf{u}}^{(i j)-}=\mathbf{X}^{(i j)+}-\mathbf{X}^{(i j)-} \text { on } \partial \omega,
\end{array}\right.
$$

with

$$
\begin{aligned}
& \mathbf{X}^{(11)+-\mathbf{X}^{(11)-}}=\left[\begin{array}{ll}
1 & 0 \\
0 & 0
\end{array}\right]\left(\mathbf{X}^{+}-\mathbf{X}^{-}\right), \\
& \mathbf{X}^{(12)+}-\mathbf{X}^{(12)-}=\left[\begin{array}{ll}
0 & 1 \\
0 & 0
\end{array}\right]\left(\mathbf{X}^{+}-\mathbf{X}^{-}\right), \\
& \mathbf{X}^{(21)+}-\mathbf{X}^{(21)-}=\left[\begin{array}{ll}
0 & 0 \\
1 & 0
\end{array}\right]\left(\mathbf{X}^{+}-\mathbf{X}^{-}\right), \\
& \mathbf{X}^{(22)+}-\mathbf{X}^{(22)-}=\left[\begin{array}{ll}
0 & 0 \\
0 & 1
\end{array}\right]\left(\mathbf{X}^{+}-\mathbf{X}^{-}\right),
\end{aligned}
$$

and $\mathbf{u}_{p}^{n l}(\mathbf{X})$ is the solution of the problem:

$$
\mathcal{L}\left(\tilde{\mathbf{u}}^{(i j)}, \boldsymbol{\delta} \mathbf{u}\right)=\mathcal{F}_{p}^{n l}(\delta \mathbf{u}) \text { in } \omega .
$$

The solution $\mathbf{u}_{p}(\mathbf{X})$ can thus be expressed by:

$$
\mathbf{u}_{p}(\mathbf{X})=\mathbb{A}(\mathbf{X}): \overline{\mathbf{F}}_{p}+\mathbf{u}_{p}^{n l}(\mathbf{X})
$$

where $\mathbb{A}(\mathbf{X})$ is a third-order tensor defined by $A_{i j k}=$ $\tilde{u}_{i}^{(j k)}$

\section{APPENDIX D.}

We expand $\mathbf{P}=\mathbf{F S}$ which leads to:

$$
\begin{aligned}
\left(\mathbf{P}_{0}+\sum_{p=1}^{N} a^{p} \mathbf{P}_{p}\right) & =\left(\mathbf{F}_{0}+\sum_{p=1}^{N} a^{p} \mathbf{F}_{p}\right) \\
& \times\left(\mathbf{S}_{0}+\sum_{p=1}^{N} a^{p} \mathbf{S}_{p}\right) .
\end{aligned}
$$


We obtain:

$$
\begin{gathered}
\mathbf{P}_{1}=\mathbf{F}_{0} \mathbf{S}_{1}+\mathbf{F}_{1} \mathbf{S}_{0} \\
\mathbf{P}_{p}=\mathbf{F}_{0} \mathbf{S}_{p}+\mathbf{F}_{p} \mathbf{S}_{0}+\sum_{i=1}^{p-1} \mathbf{F}_{i} \mathbf{S}_{p-i}, \quad \forall p>1 .
\end{gathered}
$$

By using Eq. (B.7) and setting $\sum_{i=1}^{p-1} \mathbf{F}_{i} \mathbf{S}_{p-i}=\mathbf{P}_{p}^{* n l}$, we can express $\mathbf{P}_{p}$ as:

$$
\mathbf{P}_{p}=\mathbf{F}_{0}\left\{\mathbb{C}^{(r)}: \mathbb{B}: \mathbf{F}_{p}+\mathbf{S}_{p}^{n l}\right\}+\mathbf{F}_{p} \mathbf{S}_{0}+\mathbf{P}_{p}^{* n l},
$$

which can be rewritten in the form:

$$
\mathbf{P}_{p}=\mathbb{H}^{(r)}: \mathbf{F}_{p}+\mathbf{F}_{0} \mathbf{S}_{p}^{n l}+\mathbf{P}_{p}^{* n l}
$$

Using (30), we obtain:

$$
\mathbf{P}_{p}=\mathbb{H}^{(r)}: \mathbb{A}_{, \mathbf{X}}: \overline{\mathbf{F}}_{p}+\mathbb{H}^{(r)}: \mathbf{u}_{p, \mathbf{X}}^{n l}+\mathbf{F}_{0} \mathbf{S}_{p}^{n l}+\mathbf{P}_{p}^{* n l}
$$

By setting $\mathbf{P}_{p}^{n l}=\mathbb{H}^{(r)}: \mathbf{u}_{p, \mathbf{X}}^{n l}+\mathbf{F}_{0} \mathbf{S}_{p}^{n l}+\mathbf{P}_{p}^{* n l}$ and $\mathbb{L}=$ $\mathbb{H}^{(r)}(\mathbf{X}): \mathbb{A}_{, \mathbf{X}}(\mathbf{X})$, we finally obtain:

$$
\mathbf{P}_{p}=\mathbb{L}: \overline{\mathbf{F}}_{p}+\mathbf{P}_{p}^{n l}
$$

